\title{
The thermomechanical behaviour of crosslinked polyethylene cable insulation material
}

\author{
C. P. Martin ${ }^{1}$, A. S. Vaughan ${ }^{1}$, and S. J. Sutton ${ }^{2}$ \\ ${ }^{1}$ University of Southampton, Southampton, UK \\ ${ }^{2}$ National Grid, Coventry, UK
}

\begin{abstract}
The thermomechanical properties of a practical, crosslinked EHV cable insulation material have been investigated via lathed film samples. Multiple relaxation processes are observed and discussed with regards to their effect on the short-term electrical breakdown behaviour of this material. The effect of mechanical deformation on breakdown behaviour is also investigated. It would appear that lathing induces significant damage.
\end{abstract}

\section{Introduction}

In recent years, modern plastics have been widely used as electrical insulation in high voltage power cables, gradually replacing older paper/oil systems [1]. The most favoured material is polyethylene, which exhibits excellent properties for use as a high voltage cable insulation material. However, because cable insulation operates at elevated temperatures and low density polyethylene melts at a relatively low temperature, crosslinking is generally used to improve its high temperature properties. In this paper, we set out to examine a number of fundamental issues associated with the practical use of crosslinked polyethylene (XLPE). In particular:

i) To determine the mechanical stiffness of the insulating material as a function of operating temperature.

ii) To explore the limitations placed on a cable's operating conditions by the molecular relaxation processes that operate when a high performance XLPE insulation material experiences service conditions.

iii) To examine the consequences of mechanical loading for the electrical integrity of the material.

\section{Experimental}

All the above work was performed using a practical, EHV crosslinked polyethylene cable insulation material, from which, film samples, typically $150 \mu \mathrm{m}$ in thickness, were lathed directly from the insulating layer. Dumbbell samples, die-stamped directly from the tapes were used in all subsequent mechanical tests, which were carried out with an Instron 4301 tensile testing machine equipped with a temperature control chamber. For stress-strain measurements, samples were deformed continuously to typically $200 \%$ strain over a range of temperatures. For stress relaxation tests, the samples were deformed to a fixed level of strain and allowed to relax at ambient and elevated temperatures. Because of sample variability, five tests were performed at each temperature; therefore, the data presented here constitute averages.

A Pyris DSC 7 was used to investigate the melting behaviour of these materials, via differential scanning calorimetry (DSC). The instrument was routinely calibrated with indium.

Dumbbell samples were not used in electrical tests. Instead, lathed films were deformed at ambient temperature to a fixed level of strain and allowed to relax, typically, for 48-72 hours. A standard electrical ramp test procedure was performed on each sample. The films were immersed in Dow Corning silicone oil and placed between vertically opposing $6.3 \mathrm{~mm}$ diameter ball bearing electrodes. The voltage was increased at, typically, $50 \mathrm{~V} / \mathrm{s}$ until the sample failed. This test procedure is in line with the ASTM standard D149-87.

The lathed samples were inspected via optical microscopy. A Leica DM/LM optical microscope fitted with a CCD camera was used for this purpose.

\section{Results and discussion}

\section{Tensile deformation}

Figure 1 shows the stress strain curves obtained at an extension rate of $100 \mathrm{~mm} / \mathrm{min}$. At $60{ }^{\circ} \mathrm{C}$ and below, all curves show clear evidence of multiple yielding, indicative of major structural changes within the material. These features have all but disappeared at $90{ }^{\circ} \mathrm{C}$ and above, which coincides with the melting transition for this material as observed via DSC. The Young's modulus $E$ was calculated from the initial slope of each stress-strain curve at each temperature, as shown in Fig. 2. From these data, it is evident that the material's stiffness is steadily reduced with increasing temperature up to $95{ }^{\circ} \mathrm{C}$. Above this temperature, the modulus decreases sharply, and continues to decline until a plateau is reached at $110{ }^{\circ} \mathrm{C}$. Thus, it appears that $95{ }^{\circ} \mathrm{C}$ is a sensible upper limit for the operating temperature of this system. 


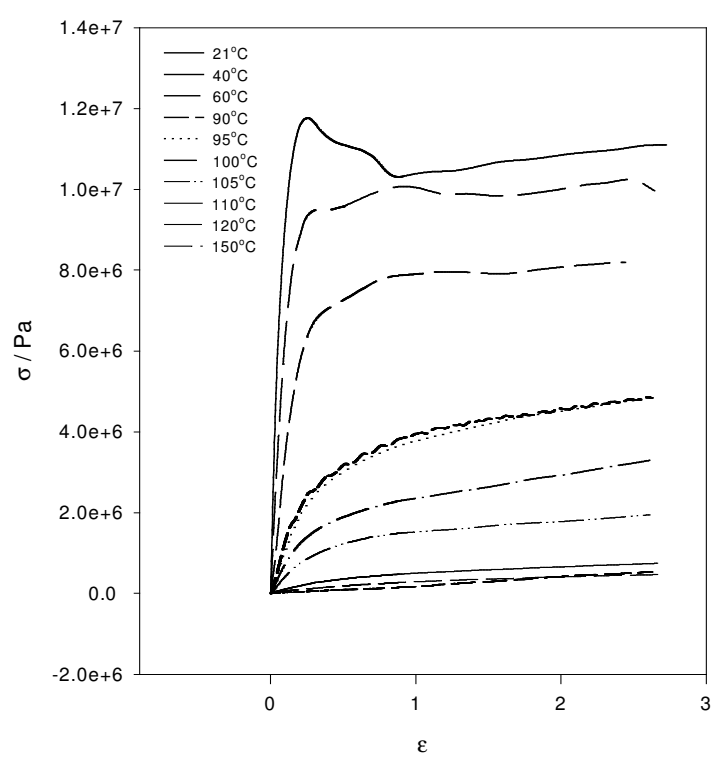

Figure 1: Isothermal stress-strain data for XLPE samples of 150 $\mu \mathrm{m}$ average thickness: $100 \mathrm{~mm} / \mathrm{min}$.

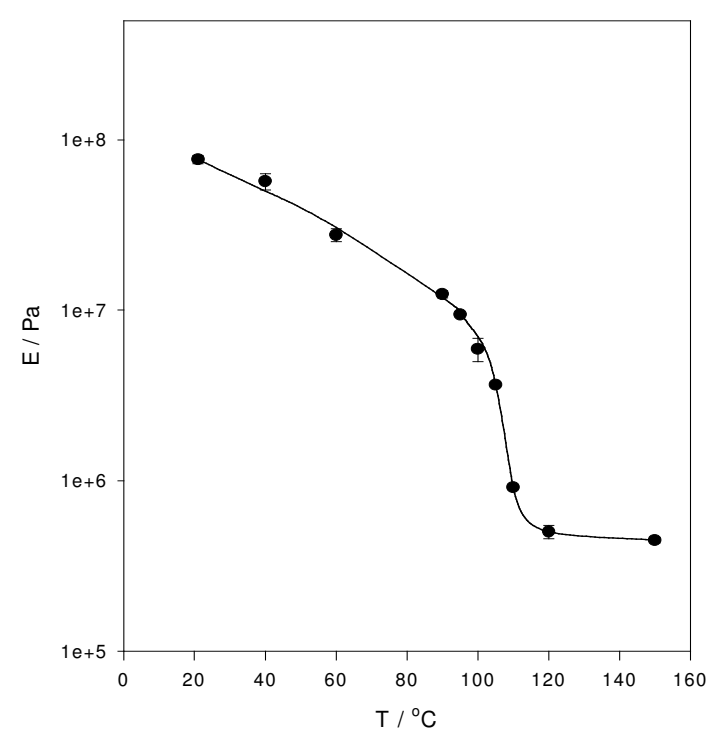

Figure 2: Young's Modulus as a function of temperature for XLPE samples.

\section{Stress relaxation}

Figure 3 shows isothermal stress relaxation curves obtained at a fixed strain of 5\%. Inspection of these data reveals that the curves do not represent a simple exponential decay, but are indicative of multiple relaxation processes at work within the material. Therefore, we decided to fit these data according to a multi-element, Maxwell-Wiechert mechanical model [2]. The individual elements in such a model correspond to single relaxation processes. It should be noted, however, that although this model is capable of describing the phenomenology of stress relaxation, it does not describe or explain the underlying physical/molecular processes. A model with multiple processes is described by (1), where $\tau$ is the relaxation time:

$$
\begin{aligned}
& \sigma(t)=\varepsilon E_{1} \exp \left(-\frac{t}{\tau_{1}}\right)+\varepsilon E_{2} \exp \left(-\frac{t}{\tau_{2}}\right)+ \\
& \varepsilon E_{3} \exp \left(-\frac{t}{\tau_{3}}\right)+\ldots . \varepsilon E_{n} \exp \left(-\frac{t}{\tau_{n}}\right)
\end{aligned}
$$

Data acquired between room temperature and $90{ }^{\circ} \mathrm{C}$ were best described by a model consisting of three Maxwell units with an offset, whereas a two unit model with an offset gave a more accurate description of the data obtained between $95{ }^{\circ} \mathrm{C}$ and $105{ }^{\circ} \mathrm{C}$. Data acquired

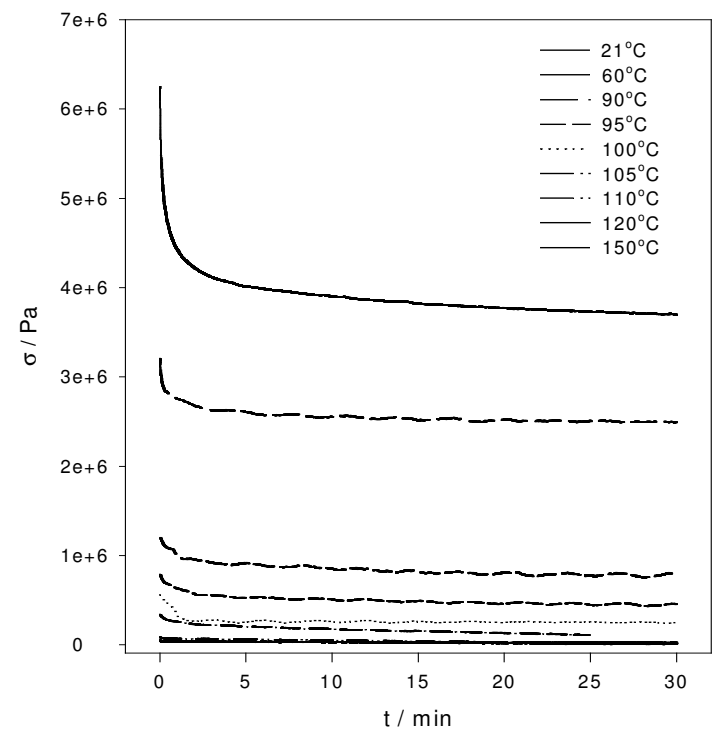

Figure 3: Isothermal stress relaxation data for XLPE samples of $150 \mu \mathrm{m}$ average thickness at $5 \%$ strain. 
between $110{ }^{\circ} \mathrm{C}$ and $150{ }^{\circ} \mathrm{C}$ were best described by a model with one Maxwell unit and an offset. Derived relaxation times are shown in Fig. 4, in which each relaxation time is associated with a single process.

From these plots, one can see clear evidence of both short and long-term relaxation processes, which successively deactivate as the temperature increases. It is believed that the processes associated with $\tau_{1}$ and $\tau_{2}$ are the so-called $\alpha_{I}$, and $\alpha_{I I}$ processes and are the dominant processes. Because of the amount of scatter in these data, one must conclude that there is no significant variation in relaxation time with temperature. This is unusual because one would expect to see an Arrheniustype temperature dependence [2], in which the relaxation time decreases exponentially with increasing temperature. There is some evidence that relaxation times can be affected in a non-Arrhenius manner by annealing effects, if the relaxations are associated with lamellar block motions [3].

It has been suggested that high temperature relaxation processes in polyethylene (i.e. $\alpha_{I}, \alpha_{I I}$ etc.) are associated with lamellar slip/deformation processes $[4,5]$. It is also interesting to note that yielding behaviour appears to cease at the same temperatures that the short-term processes become inactive. In addition, DSC studies have revealed that levels of crystallinity within the material decline significantly above $95{ }^{\circ} \mathrm{C}$.

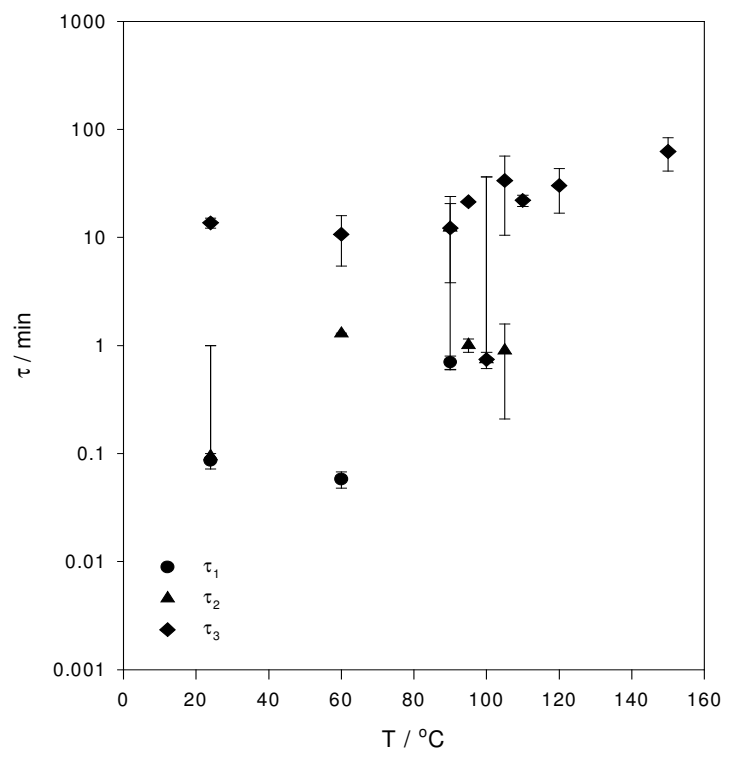

Figure 4: Relaxation times as a function of temperature for XLPE samples: $5 \%$ strain.
These observations would therefore seem to provide strong evidence that yielding and short-term relaxation phenomena both originate within the lamellar regions of this material. The third relaxation process, associated with $\tau_{3}$, operates over a longer timescale than the other two and does not appear to correlate with yielding or crystallinity. Because of this, it is most likely that this third process is best associated with the crosslinked network. Further experiments were conducted at a fixed strain of $100 \%$ but did not produce significantly different results from those already described.

\section{Electrical breakdown}

During the initial relaxation studies, samples, typically $150 \mu \mathrm{m}$ in thickness, were deformed to $100 \%$ strain at ambient temperatures and left to relax for between 16 and $86 \mathrm{hr}$. It was found that the mean breakdown strength did not vary appreciably over the measured time period. Because the relaxation times display only a weak dependence upon temperature, one might therefore expect this effect to be valid over the range of operating temperatures for this material. Undeformed samples of the same thickness were found to possess a mean breakdown field strength of $139 \mathrm{kV} / \mathrm{mm} \pm$ $7 \mathrm{kV} / \mathrm{mm}$ (the $95 \%$ confidence interval).

Figure 5 shows the mean breakdown strength of XLPE tape samples as a function of deformation, after being allowed to relax for at least 72 hours. All sample thicknesses were initially $150 \mu \mathrm{m}$. From these data, one can observe that $\mathrm{i}$ ), there is a large amount of scatter and ii) no clear trends are visible. These results are surprising as, on the basis of electrical treeing work on XLPE and ramp tests of laboratory-prepared samples, mechanical stresses should produce a significant effect $[6,7]$. However, one must bear in mind that there is a large amount of scatter in these data, which may be masking these effects. The samples used in this study were not well-defined laboratory systems; i.e. they were lathed directly from an EHV cable. This is a damaging process, as can clearly be seen in Fig .6, which shows the surface of an untested, lathed XLPE sample. These irregularities would lead to localized field enhancements and, hence, reduce the breakdown strength across the solid/oil interface [8]. Also, this is most likely the origin of the large amount of scatter in these data. It is also our belief that many of the electrical effects associated with mechanical deformation and relaxation are rather subtle in nature, even for well-defined model systems [7]. It is likely that deformation induced effects would only become noticeable if unrealistic strains were used. We are therefore forced to conclude that, in isolation, the lathing of thin film samples from XLPE cables is not ideal for tests of this nature. 


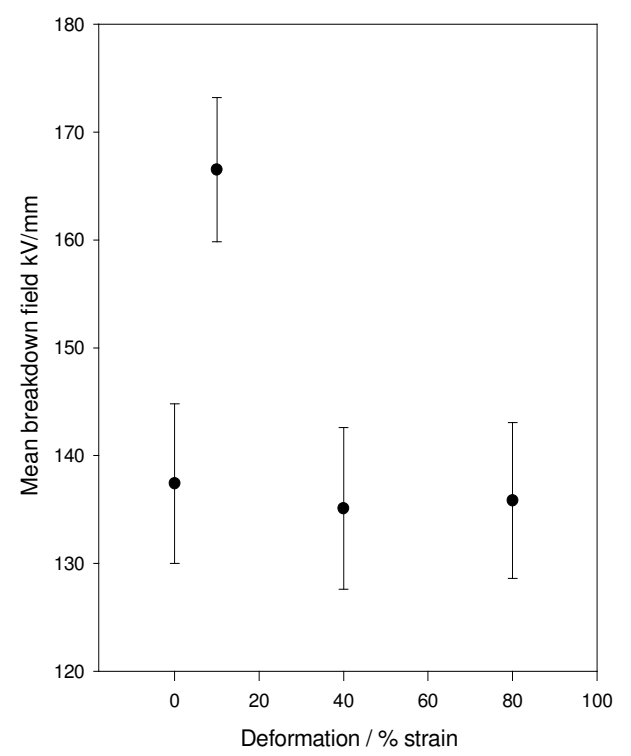

Figure 5: Mean breakdown field strength as a function of deforrmation at ambient temperature.

\section{Conclusions}

We have investigated the thermomechanical and electrical properties of a high performance XLPE electrical insulation material. This was found to maintain a good level of mechanical stiffness up to $95^{\circ} \mathrm{C}$. Relaxation measurements have shown that there are two main, short-term processes that operate below this temperature and a third process that operates over the entire temperature range. The former are probably associated with crystalline regions within this material, as are the yield points which appear within the stressstrain data, while the latter is best associated with the crosslinked network. An attempt was made to investigate the electrical breakdown behaviour as a function of deformation and relaxation time. Relaxation was observed to have little discernable effect on the short-term breakdown behaviour of this material, as was the degree of deformation. The latter result was attributed to surface damage suffered during lathing.

\section{Acknowledgements}

The authors acknowledge the support of The National Grid Company plc and their permission to publish this work.

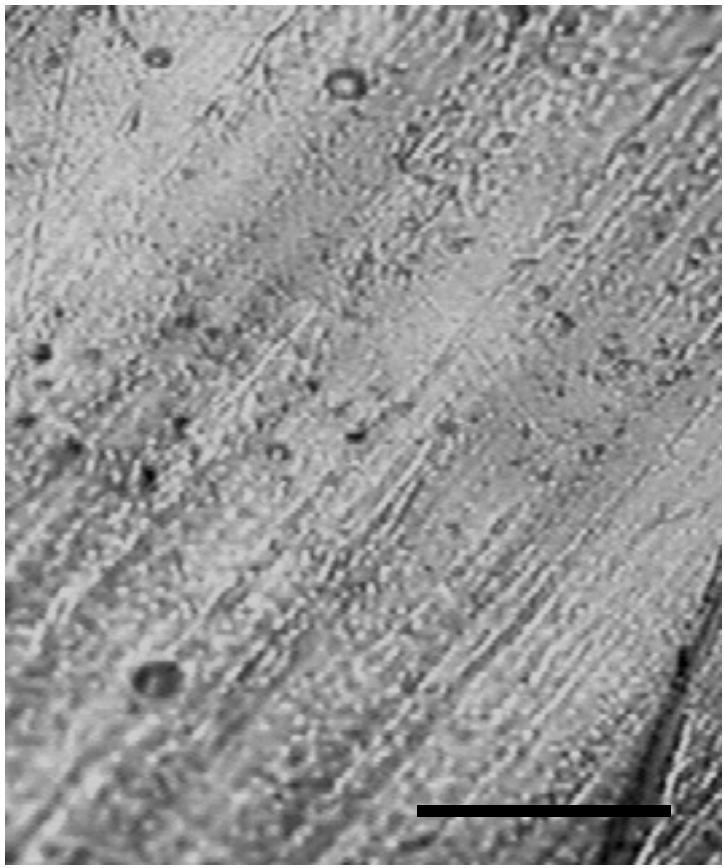

Figure 6: Optical micrograph showing the surface damage inflicted on an XLPE sample by the lathing process. Scale bar $40 \mu \mathrm{m}$.

\section{References}

[1] G. Greenway, The Effect of Processing Conditions on the Morphology and Electric Strength of Polyethylene Blends, $\mathrm{PhD}$ thesis, University of Reading 2000.

[2] I. M. Ward, D. W. Hadley, An Introduction to the Mechanical Properties of Solid Polymers, Wiley, Chichester:UK, 1993.

[3] R. J. Young, Introduction to Polymers, Chapman and Hall, 1989

[4] A. R. Plaza, E Ramos, A Manzur, R Olavo and A Escobar, "Double Yield Points in Triblends of LDPE, LLDPE and EPDM", J. Mater. Sci., vol. 32, pp. 549-554. Jan. 1997.

[5] N. W. Brooks, R. A. Duckett and I. M. Ward, "Investigation into Double Yield Points in Polyethylene", Polymer, vol. 33, pp. 1872-1886.

[6] E. David, J. L. Parpal and J. P. Crine, "Influence of Internal Mechanical Stress and Strain on Electrical Performance of Polyethylene Electrical Treeing Resistance", IEEE Trans. Diel Elec. Eng, vol. 3, pp. 248-257. Apr. 1996.

[7] I. L. Hosier, Morphology and Electrical Properties of Polyethylene Blends, PhD thesis, University of Reading 1996.

[8] A. M. S. Katahoire, M. R. Raghuveer and E. Kuffel, "Power Frequency and Impulse Voltage Breakdown along Silicone Oil / XLPE Interface", IEEE Trans. Elec. Eng, vol. 15, pp. 97-104. Apr. 1980.

Author address: Christopher P. Martin, ECS, Faraday Building, University of Southampton, Southampton SO17 1BJ, UK, Email: cpm00r@ecs.soton.ac.uk 